\title{
Supporting Information - Line Waves Existing at Junctions of Dual-Impedance Metasurfaces
}

\author{
Zhixia Xu ${ }^{1,2}$, Jie Chang ${ }^{1}$, Shaojun Fang ${ }^{1}$, Qiuyi Zhang ${ }^{2}$, Robert J. Davis ${ }^{3}$, Dan Sievenpiper ${ }^{3}$, and
} Tie Jun Cui ${ }^{2}$

${ }^{1}$ School of Information Science and Technology, Dalian Maritime University, Dalian 116026, China.

${ }^{2}$ State Key Laboratory of Millimeter Waves, Southeast University, Nanjing 210096, China.

${ }^{3}$ Electrical and Computer Engineering Department, University of California San Diego, San Diego, California 92093, USA.

\section{Dispersion and equivalent impedance of unit cells}

We start from the modeling and simulation of the unit cell. We first open the CST Studio Suite and select the eigenmode solver. The models of unit cell are shown in Fig. S1(a) and (b). The boundaries along $x$ direction and $y$-direction are set as periodic boundary condition, close to the structure. The boundaries along $z$-direction are set as electric boundary condition, more than $\lambda / 4$ away from the structure and the sufficient distance can preclude the errors caused by boundaries to surface modes under study. The frequency range is set from $0 \mathrm{GHz}$ to $40 \mathrm{GHz}$. Then, we set parameter sweep of the phase shift along $x$ direction from 0 degree to 180 degrees and finally get the dispersion curves of the unit cells, as shown in Fig. 1(b) and (e) in the main manuscript.

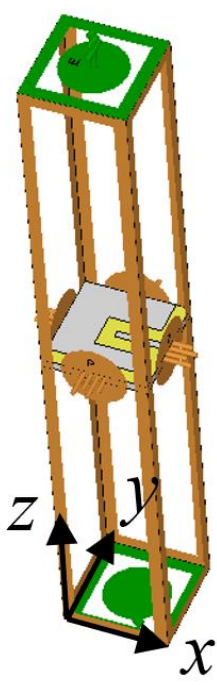

(a)

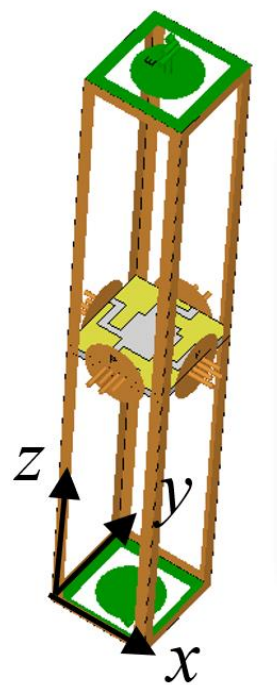

(b)

Fig. S1. (a) Detailed structure of the proposed inductive unit cell. (b) Detailed structure of the proposed capacitive unit cell.

Using Eq. (S1) and Eq. (S2), we can calculate the equivalent impedance of inductive and capacitive unit cells from simulated dispersion curves. In a new simulation model, we make a flat sheet $\mathrm{Z}_{\mathrm{TM}}$ and change the material type from normal to surface impedance and import the calculated impedance table, as shown in Fig. S2(a) and (b). Following the same steps, we make a flat sheet $Z_{\mathrm{TE}}$, as shown in Fig. S2(c) and (d). 
The fitting curves of equivalent surface impedances in CST Studio Suite are shown in Fig.S3(a) and (b). Therefore, we can use $\mathrm{Z}_{\mathrm{TM}}$ and $\mathrm{Z}_{\mathrm{TE}}$ flat sheets as substitutes for the complex inductive and capacitive unit cells and further compose the impedance interface. The simplified 1-layer and 2-layer structures are shown in Fig.S4(a) and (b), the boundaries are set as open (add space) boundaries.

$$
\begin{aligned}
& Z_{\mathrm{TM}}=\eta_{0} \sqrt{1-\frac{k_{t}^{2}}{k_{0}^{2}}} \\
& Z_{\mathrm{TE}}=\eta_{0} / \sqrt{1-\frac{k_{t}^{2}}{k_{0}^{2}}}
\end{aligned}
$$

where $k_{t}$ is the wavevector along the surface, $k_{0}$ is the wavevector in vacuum, and $\eta_{0}$ is the surface impedance in vacuum.

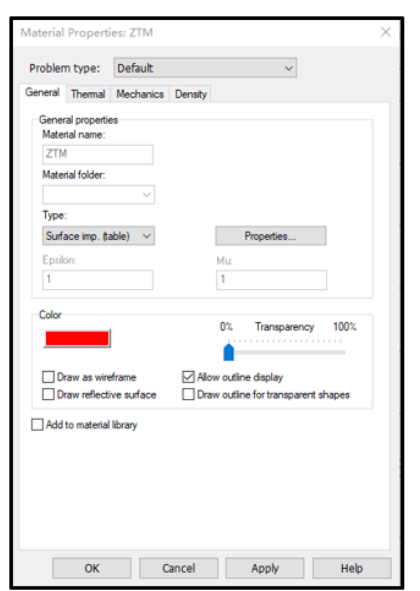

(a)

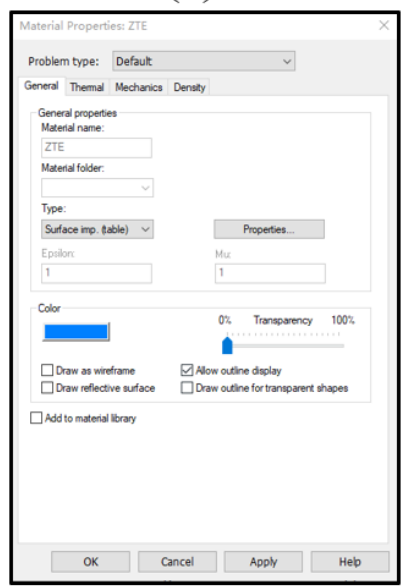

(c)

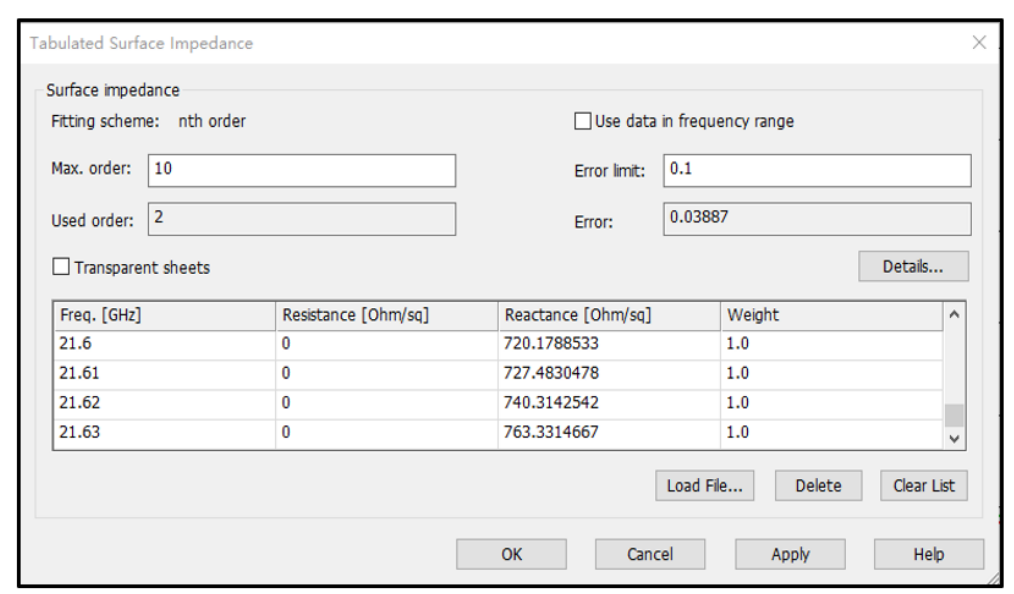

(b)

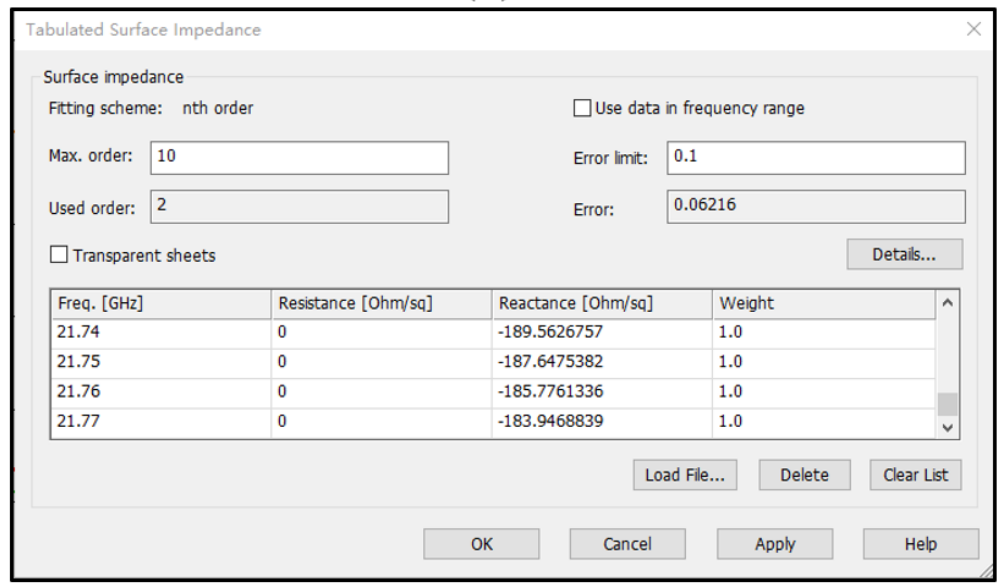

(d)

Fig. S2. Equivalent inductive surface set up: (a) material properties, (b) imported surface impedance. Equivalent capacitive surface set up: (c) material properties, (d) imported surface impedance. 


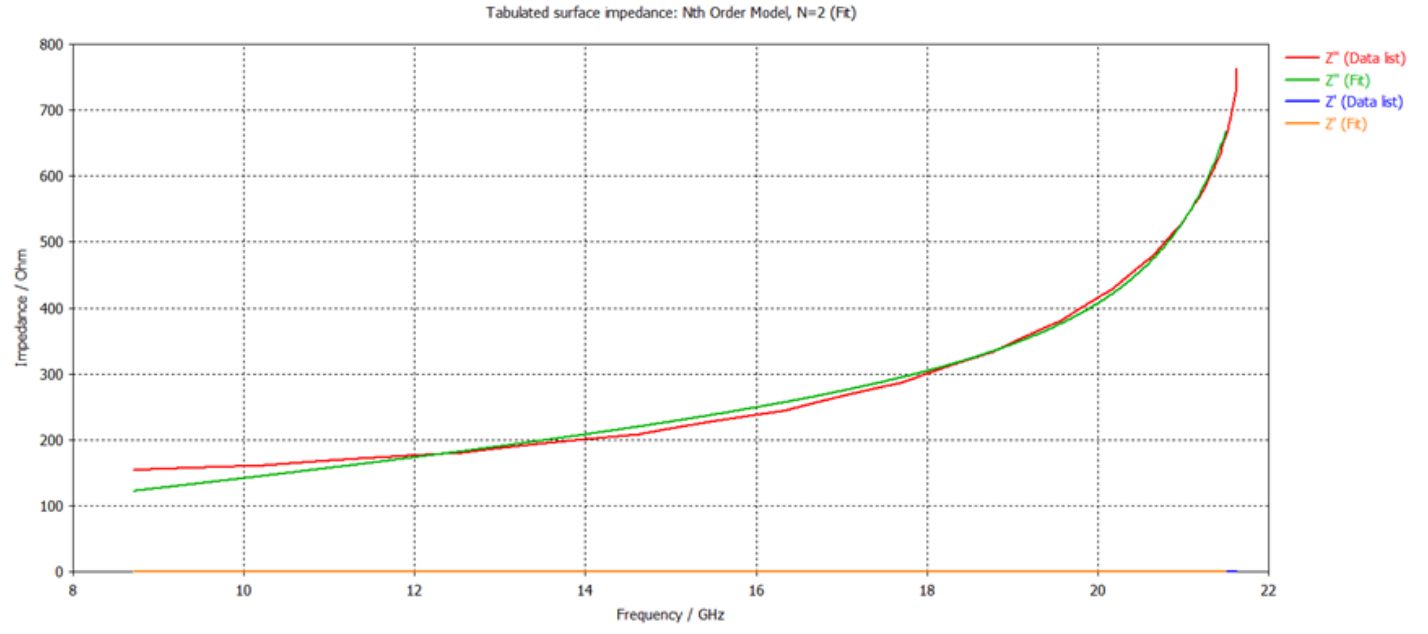

(a)

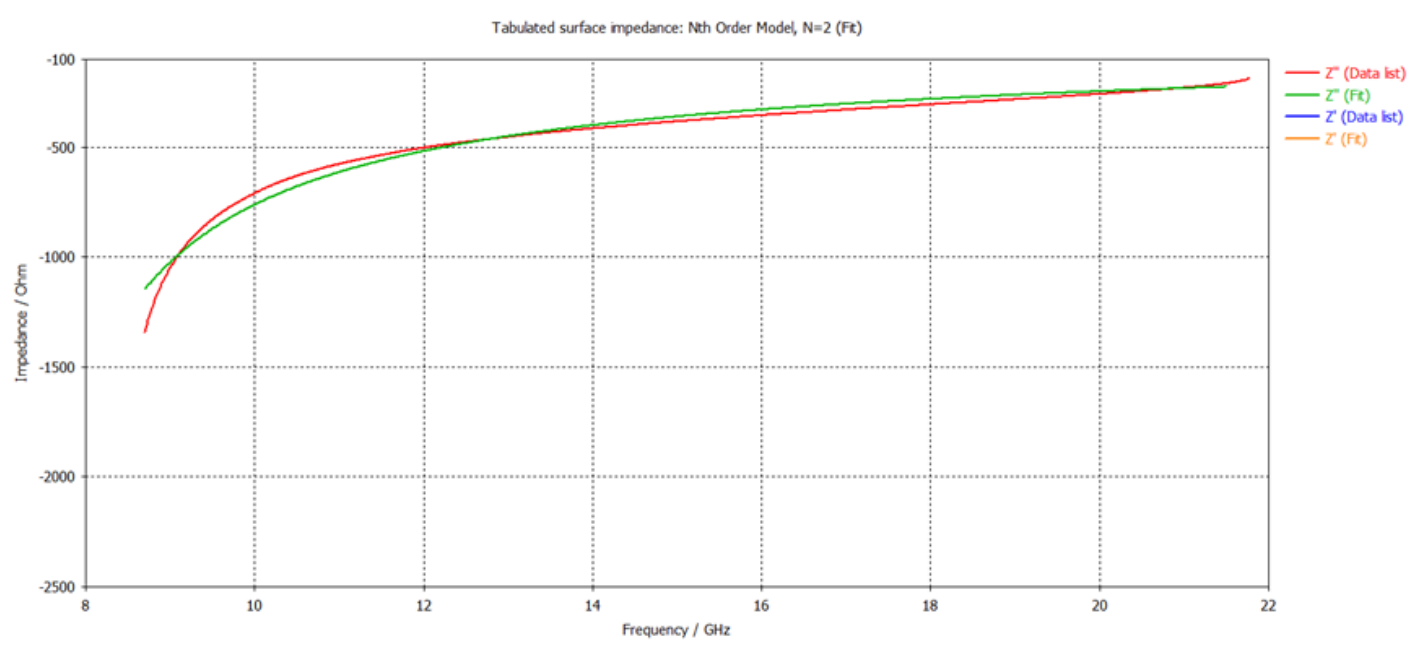

(b)

Fig. S3. (a) The fitting curve of the inductive surface. (b) The fitting curve of the capacitive surface.

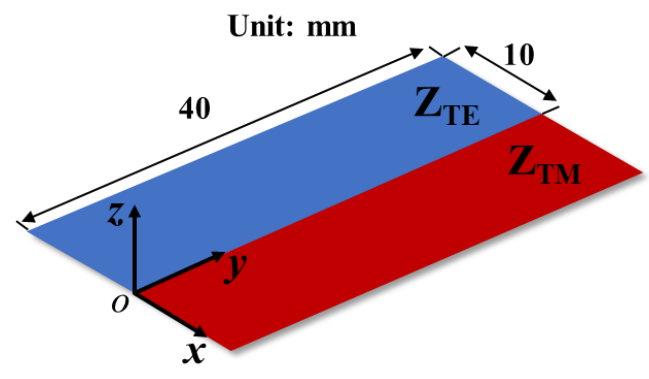

(a)

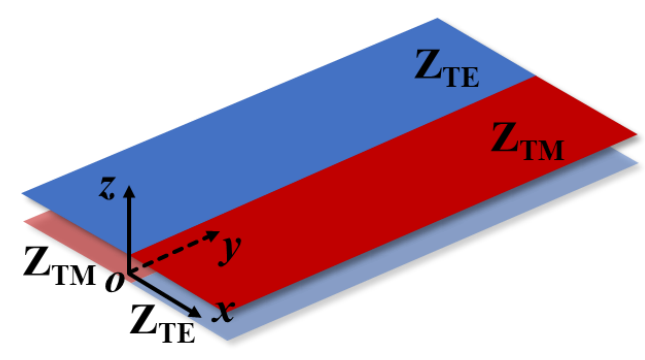

(b)

Fig. S4. (a) The 1-layer structure based on equivalent impedance surfaces. (b) The 2-layer structure based on equivalent impedance surfaces. 


\section{Dispersion of line waves}

In this section, we introduce the simulation of dispersion of line waves supported by the 2-layer structure shown in Fig. S5. The boundaries along $x$-direction are set as periodic boundary condition, close to the structure. The boundaries along $y$-direction are set as magnetic boundary condition, close to the structure. The boundaries along $z$-direction are set as electric boundaries, more than $\lambda / 4 \mathrm{~mm}$ away from the structure. The frequency range is set from $0 \mathrm{GHz}$ to $40 \mathrm{GHz}$. Using the eigenmode solver of CST Studio Suite, we can get the dispersion curves of line waves, as shown in Fig. 5(a) and (d) in the manuscript.

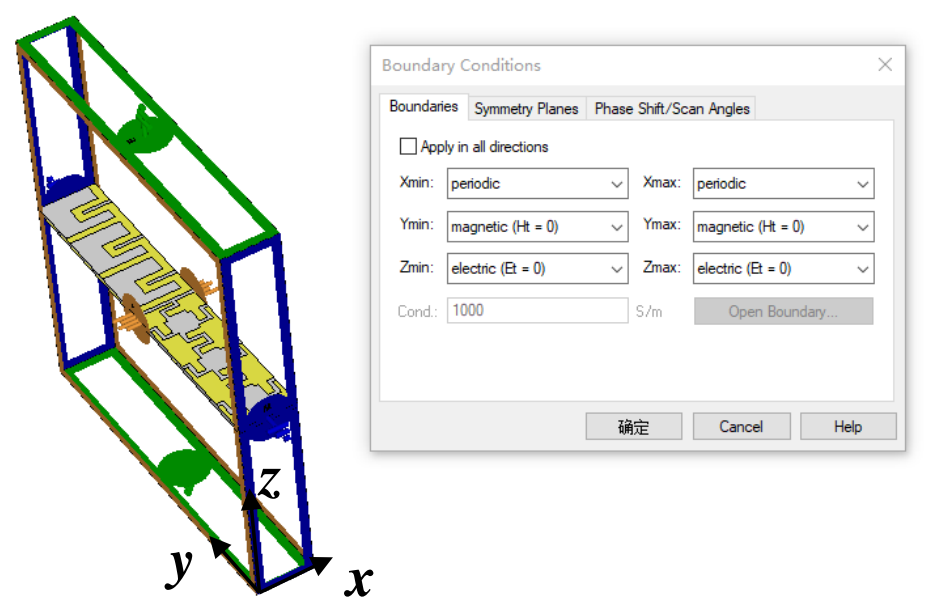

Fig. S5. Simulation model of the 2-layer superlattice.

\section{Parallel-plate metasurface waveguide}

In this section, we introduce the simulation of the 2-layer metasurface waveguide with 40 periodic unit cells along the $x$-direction. The model is excited by parallel transmission lines connected with two $\mathrm{Z}_{\mathrm{TM}}$ metasurfaces on top and bottom layers directly, as shown in Fig. S6. Two wave ports at terminals are set to calculate the transmission efficiency. Open (add space) boundaries are set out of the full model to simulate the situation where the model is placed in the free space. The frequency range is from $0 \mathrm{GHz}$ to $40 \mathrm{GHz}$. We use the time domain solver. The simulated results are shown in Fig. 5 and 6 of the manuscript.

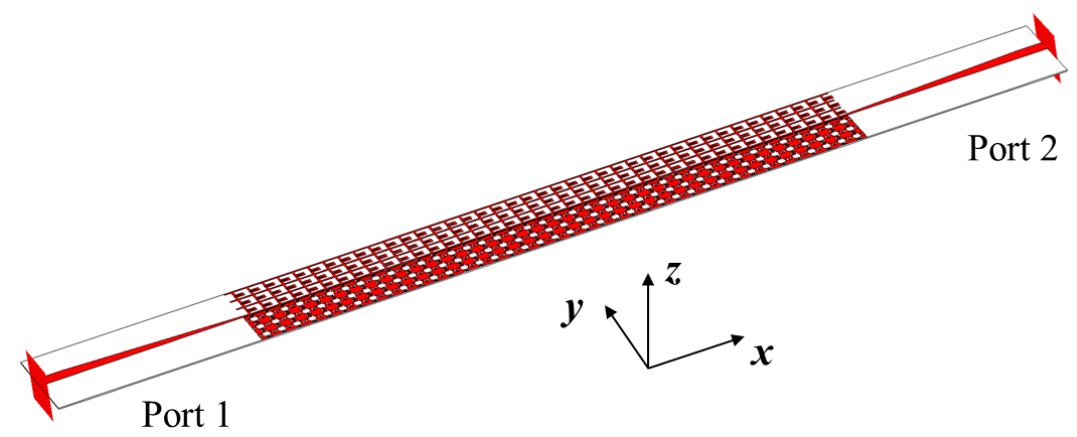

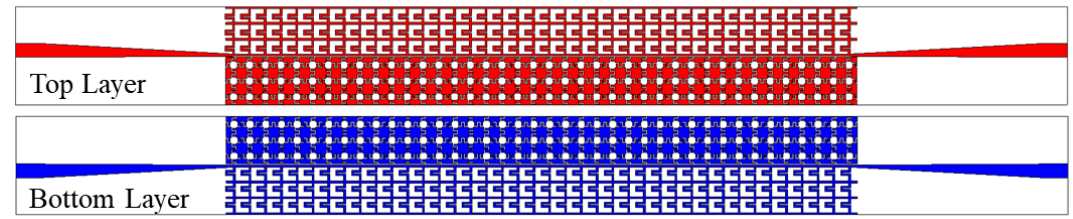

Fig. S6. The 2-layer metasurface waveguide consisting of four metasurfaces arranged with duality. 


\section{Spin-momentum locking}

In this section, we introduce the simulation of chiral sorting of line waves excited by circular-polarized (CP) dipoles placed beside the junction. Two dipole antennas excited by discrete port 1 and port 2 with phase difference of 90 degrees are used to generate the CP field. The frequency range is set from 8.7 $\mathrm{GHz}$ to $21.5 \mathrm{GHz}$ and open (add space) boundaries are used. The simulation model is shown in Fig. S7. We add electric field monitors to observe electric field distributions. By post-processing, we combine the electric field excited by two ports to analyze the chiral sorting of opposite CP source. Settings in CST Studio Suite are shown in Fig. S8, where $\pm \pi / 2$ phase difference between port 1 and port 2 decides the handedness of the source. Simulated results are shown in Fig. 3 and 4 of the manuscript.

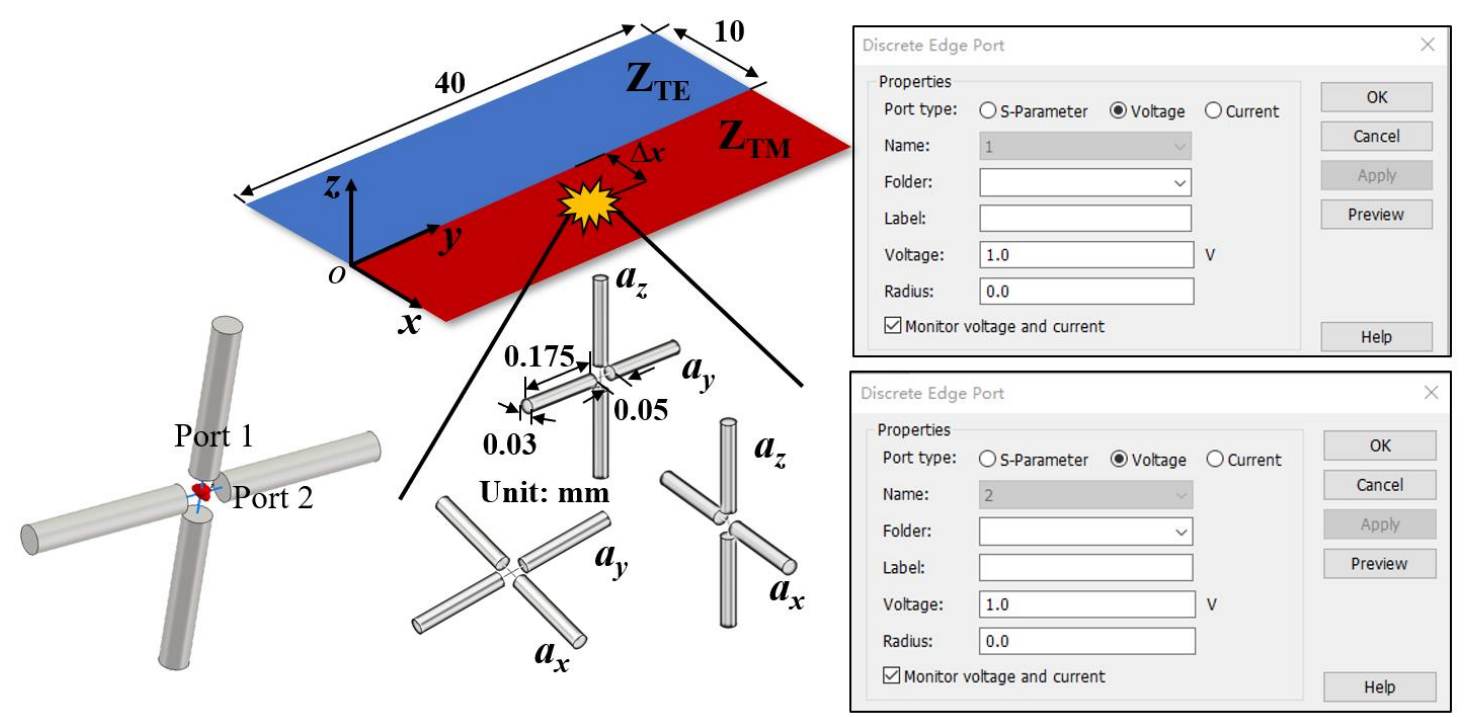

Fig. S7. Chiral sorting based on different configurations of CP dipoles.

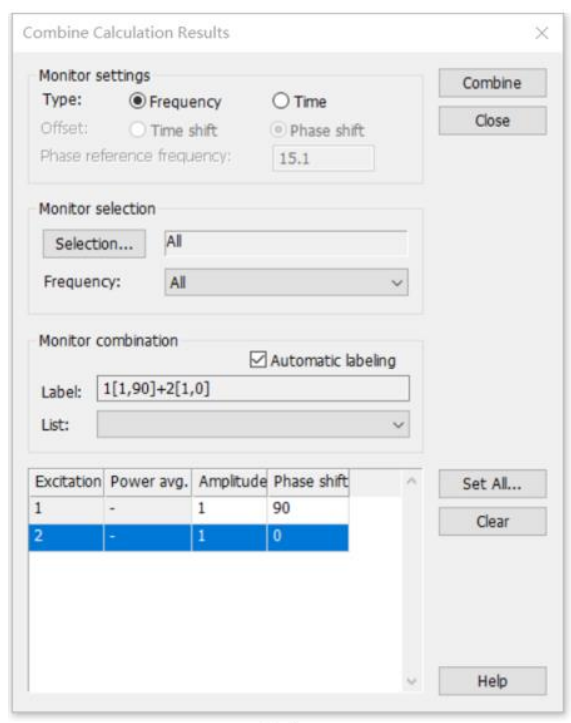

(a)

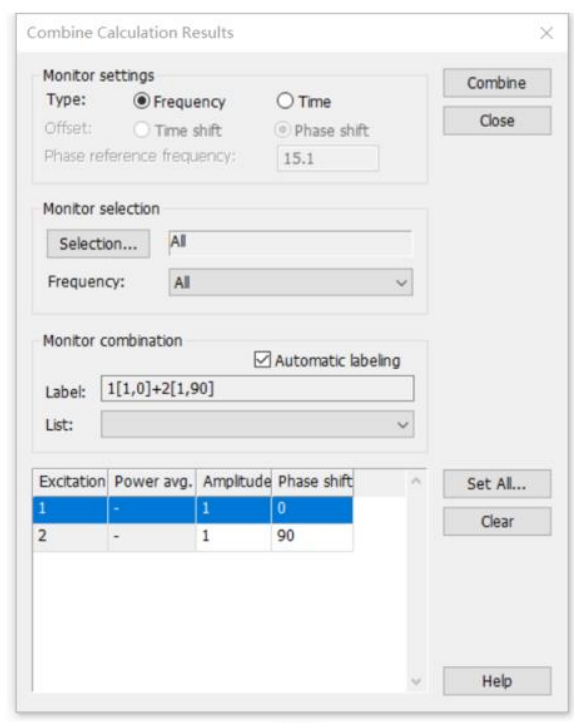

(b)

Fig. S8. The combination of results using CST post-processing function: (a) Port 1 is set with 90degrees ahead. (b) Port 2 is set with 90-degrees ahead. 